\title{
Open Flow Microperfusion as a Dermal Pharmacokinetic Approach to Evaluate Topical Bioequivalence
}

\author{
Manfred Bodenlenz ${ }^{1}$ Katrin I. Tiffner ${ }^{1} \cdot$ Reingard Raml $^{1} \cdot$ Thomas Augustin $^{1}$ • \\ Christian Dragatin ${ }^{1}$ - Thomas Birngruber ${ }^{1} \cdot$ Denise Schimek $^{1} \cdot$ Gerd Schwagerle $^{2}$. \\ Thomas R. Pieber ${ }^{1,2} \cdot$ Sam G. Raney $^{3} \cdot$ Isadore Kanfer $^{4,5} \cdot$ Frank Sinner $^{1,2}$
}

Published online: 18 August 2016

(c) The Author(s) 2016. This article is published with open access at Springerlink.com

\begin{abstract}
Background The availability of generic topical dermatological drug products is constrained by the limited methods established to assess topical bioequivalence (BE). A novel cutaneous pharmacokinetic approach, dermal open-flow microperfusion (dOFM), can continuously assess the rate and extent to which a topical drug becomes available in the dermis, to compare in vivo dermal bioavailability (BA) and support BE evaluations for topical products.

Objective To evaluate whether dOFM is an accurate, sensitive, and reproducible in vivo method to characterize the intradermal BA of acyclovir from $5 \%$ acyclovir creams, comparing a reference $(R)$ product either to itself or to a different test $(T)$ product.

Methods In a single-center clinical study, $R$ or $T$ products were applied to six randomized treatment sites on the skin of 20 healthy human subjects. Two dOFM probes were inserted in each treatment site to monitor the intradermal acyclovir concentration for $36 \mathrm{~h}$. Comparative BA (of $R$ vs.
\end{abstract}

Frank Sinner

frank.sinner@joanneum.at

1 HEALTH-Institute for Biomedicine and Health Sciences, Joanneum Research Forschungsgesellschaft $\mathrm{mbH}$, Neue Stiftingtalstrasse 2, 8010 Graz, Austria

2 Division of Endocrinology and Diabetology, Department of Internal Medicine, Medical University of Graz, Graz, Austria

3 Division of Therapeutic Performance, Office of Research and Standards, Office of Generic Drugs, US Food and Drug Administration, Silver Spring, MD, USA

4 Faculty of Pharmacy, Rhodes University, Grahamstown, South Africa

5 Present Address: Leslie Dan Faculty of Pharmacy, University of Toronto, Toronto, ON, Canada
$R$ and $T$ vs. $R$ ) was evaluated based on conventional BE criteria for pharmacokinetic endpoints (area under the curve and maximum plasma concentration) where the $90 \%$ confidence interval of the geometric mean ratio between the $T$ and $R$ falls within $0.80-1.25$.

Results The positive control products $(R$ vs. $R$ ) were accurately and reproducibly confirmed to be bioequivalent, while the negative control products ( $T$ vs. $R$ ) were sensitively discriminated not to be bioequivalent.

Conclusions dOFM accurately, sensitively, and reproducibly characterized the dermal BA in a manner that can support BE evaluations for topical acyclovir $5 \%$ creams in a study with $n=40$ (20 subjects in this study).

\section{Key Points}

This is the first study showing the utility of clinical dermal open-flow microperfusion (dOFM) as a dermal pharmacokinetic approach to compare dermal bioavailability (BA) and support bioequivalence (BE) evaluations for a topical (locally acting) drug product.

dOFM is capable of directly measuring the penetration of topically applied acyclovir in human subjects in vivo with low variability for prolonged durations.

dOFM has the necessary accuracy and reproducibility to confirm $\mathrm{BE}$ for a reference acyclovir cream $5 \%$ compared with itself, and is sufficiently sensitive to discriminate inequivalent BA between two different topical acyclovir cream $5 \%$ products, in both cases based upon conventional BE criteria and pharmacokinetic endpoints. 


\section{Introduction}

In many countries, strategies to control healthcare spending rely upon the availability and use of generic medicines. The safety and effectiveness of high-quality generic medicines is ensured through a demonstration of bioequivalence (BE) [1]. The methodology for assessing the BE of systemically absorbed drugs and for the statistical assessment of comparative systemic bioavailability (BA) based upon pharmacokinetic (PK) endpoints is well established. The same is not true for topical drug products, which are not intended to be absorbed into the systemic circulation. In the former instance, conventional regulatory $\mathrm{BE}$ acceptance criteria are based on the maximum plasma concentration $\left(C_{\max }\right)$ of the drug and the area under the curve (AUC) of the plasma drug concentration vs. time profiles. For pharmaceutically equivalent products to be considered bioequivalent, the $90 \%$ confidence interval for the ratio of the means of each of these PK endpoints should fall within specified BE limits (typically $0.80-1.25$ ) on a $\log$ transformed basis [2, 3]. For topical glucocorticoid drug products, the vasoconstrictor assay is a well-established approach to efficiently evaluate BE [4]; however, it has limited scope only to that class of drug products. Otherwise, in general, a randomized, double-blind, parallel, placebo-controlled, comparative, clinical endpoint BE study that compares the test (generic) product with the reference listed drug product is the default approach routinely accepted in USA to demonstrate BE for most locally or regionally acting topical dosage forms [5]. This impacts the availability of topical generic products because such clinical endpoint BE studies may require even more participants than the studies originally performed by the innovator for the new drug [6]. Moreover, clinical endpoint BE studies may not be the most accurate, sensitive, or reproducible approach by which to distinguish differences in BA between different topical drug products $[6,7]$. While a cutaneous PK approach may be a more efficient way to assess $\mathrm{BE}$, evaluating the rate and extent at which the topical drug becomes available at the site of action (in the viable epidermis and dermis) is particularly challenging. Different recommendations for such BE evaluations have previously been suggested [8-10].

To address this issue and help make high-quality topical generic drug products available to patients, Raney et al. explored the possibility of developing appropriate PKbased $\mathrm{BE}$ approaches for the comparative assessment of BA from topical dermatological drug products [11]. Evidence supporting the potential feasibility of such alternative in vivo methods has been presented in the literature, notably relating to tape stripping [12-14] and dermal microdialysis [11, 15-21].

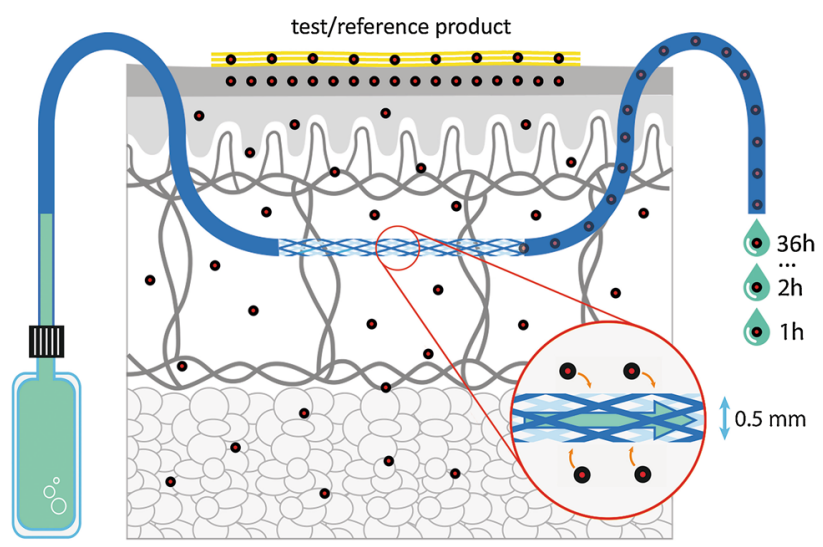

Fig. 1 Schematic of dermal open flow microperfusion

More recently, dermal open-flow microperfusion (dOFM) has been investigated as another potential approach to assess the $\mathrm{BA} / \mathrm{BE}$ of topical products. It facilitates a continuous assessment of the in vivo cutaneous kinetics of topically administered drugs directly in the dermis in human subjects. dOFM can assess the intradermal biochemistry and drug concentrations by sampling the dermal interstitial fluid for up to $48 \mathrm{~h}$ (Fig. 1). The benefit of dOFM has been shown by PK-pharmacodynamic studies where a wide range of substances from small lipophilic drugs to large proteins and antibodies could be successfully monitored in the dermis of healthy human subjects and patients [22-25]. Additionally, dOFM may be used with patients experiencing dermatologic diseases and may enable evaluation of topical BE under conditions where the disease status of the skin barrier may lead to altered BA of the drug.

The overall aims of this study were (1) to evaluate whether dOFM can accurately and reproducibly identify the in vivo BA of acyclovir in the dermis to be equivalent between two treatment sites both dosed with the same $R$ acyclovir cream $5 \%\left(R_{2}\right.$ vs. $\left.R_{1}\right)$ and (2) to evaluate whether dOFM has the sensitivity to discriminate the in vivo BA of acyclovir in the dermis as not bioequivalent (based upon conventional BE criteria) between two treatment sites dosed with compositionally different acyclovir cream $5 \%$ products ( $T$ vs. $R_{1}$ ) marketed in different countries.

\section{Materials and Methods}

\subsection{Healthy Subjects}

Twenty healthy human subjects were enrolled (Caucasian, 7 women, 13 men, age $28 \pm 5$ years). 


\subsection{Topical Study Drugs}

Acyclovir cream $5 \%$ (Zovirax $^{\circledR}$; Valeant, Bridgewater, NJ, USA) was used as the $R$ product. Acyclovir cream $5 \%$ (Aciclovir 1A Pharma-Creme; 1A Pharma GmbH, Vienna, Austria), which has a different formulation composition and from which the topical BA of acyclovir was observed to be different relative to the $R$ product (unpublished results from exploratory, pilot, in vivo dOFM studies), was used as the $T$ product.

\section{3 dOFM}

dOFM probes were inserted intradermally (two probes at each of the three treatment sites on each limb) and dermal interstitial fluid samples were continuously collected $(1 \mu \mathrm{L} / \mathrm{min})$ from a pre-dose baseline time period $(-1 \mathrm{~h}$ to $0 \mathrm{~h}$ ) to $36 \mathrm{~h}$ post-dose. The dOFM devices (sterile probes, wearable pumps, accessories; CE certified for human use) were developed by HEALTH-Joanneum Research GmbH (Graz, Austria). The devices and their clinical use have been described in detail previously [23, 24]. In this study, the newest version of a CE-certified dOFM probe (DEA15003) was used. Like its precursors, this new dOFM probe is a highly flexible linear probe with a demarcated $15-\mathrm{mm}$ fully permeable section, and it also has imprinted position markers to facilitate the precise positioning of the permeable section of the probe below the treatment area. The probe's outer diameter is $0.5 \mathrm{~mm}$ and it is inserted over a length of $30 \mathrm{~mm}$ using a standard $0.9-\mathrm{mm}$ (outer diameter) hollow insertion needle.

\subsection{Study Design}

After enrollment and qualification of study subjects based upon the protocol inclusion and exclusion criteria, a set of three treatment sites (referred to as the 'test triad') was demarcated on each thigh as depicted in Fig. 2 (providing a total of six treatment sites per subject, each $\left.5.5 \mathrm{~cm}^{2}\right)$. Twelve dOFM probes were inserted intradermally (two replicate probes per treatment site) and dermal interstitial fluid was continuously sampled at $1 \mu \mathrm{L} / \mathrm{min}$ using sterile perfusate that included $1 \%$ albumin and $600 \mathrm{mg} / \mathrm{dL}$ glucose. The skin at a treatment site was cooled using a sterile ice bag prior to probe insertion as well as during the initial hour following probe insertion.

At baseline, the transepidermal water loss was measured on the skin of each leg (Aquaflux AF200; Biox Ltd, London, UK) and the baseline serum and dOFM samples were collected.

At $t=0,15 \mathrm{mg}$ cream $/ \mathrm{cm}^{2}$ of each of the $R$ and $T$ products were applied to the respective treatment sites on the skin using a tared spatula. The spreading procedure was of standardized duration ( $1 \mathrm{~min}$ ) for all treatment sites and care was taken to consistently dose the cream as a homogenous layer. The $R$ product was applied at two sites on each thigh $\left(R_{1}\right.$ central, $R_{2}$ non-central) and $T$ non-central (Fig. 2a) in a randomized order, using treatment randomization sequences of either ' $R_{2}-R_{1}-T$ ' or ' $T-R_{1}-R_{2}$ ' according to a randomization scheme that had been predefined in the study protocol to rule out the impact of anatomical location or procedural factors. Treatment sites were protected by a transparent, non-occlusive, domeshaped, perforated plastic shield (Fig. 2b) and samples of the continuous perfusion from the dOFM probes in the dermis were collected at 4-h intervals, up to $36 \mathrm{~h}$ postdose. Glucose in dOFM samples was measured at the bedside (Super GL; Dr. Müller Gerätebau GmbH, Freital, Germany) and the relative glucose loss from the perfusate (relative to $600 \mathrm{mg} / \mathrm{dL}$ in \%) was calculated to monitor the exchange rate ('relative recovery') across the probe.

Study subjects rested in bed most of the time and slept in a supine position during the night. The sampling procedure during the night was identical to that used during the day. After termination of the study at $36 \mathrm{~h}$, the intradermal position of each probe was assessed by a longitudinal ultrasound scan (GE LOGIQ e R6 device with linear $22 \mathrm{MHz}$ probe; GE Healthcare, Vienna, Austria).

To reduce perturbations to the probes and to minimize variability in the kinetic data, any torsional strains or skin stretching from anatomical flexion at the test triads across $36 \mathrm{~h}$ were minimized through the use of self-adhesive stabilization rings (HEALTH-Joanneum Research GmbH). As additional controls to reduce experimental variability and optimize the precision and reproducibility of the study, room temperature and relative humidity were tightly controlled throughout the study $\left(22 \pm 1{ }^{\circ} \mathrm{C}, 40-60 \%\right.$ relative humidity).

\subsection{Sample Analyses}

Acyclovir determination: Samples $(20 \mu \mathrm{L}$ of perfusate plus D4-deuterated acyclovir internal standard) were processed by solid-phase extraction (Oasis MCX $\mu$ Elution plate; Waters, Milford, MA, USA). Samples were washed with formic acid/water (5\%/95 \%, v/v) followed by methanol; eluted with $\mathrm{NH}_{4} \mathrm{OH} /$ methanol (5\%/95 \%, v/v); evaporated to dryness; and finally reconstituted in $20 \mu \mathrm{L}$ methanol/ water $(5 \% / 95 \%, \mathrm{v} / \mathrm{v})$. High-performance liquid chromatography (HPLC) analysis of acyclovir in dOFM samples was performed with an Ultimate 3000 HPLC (Thermo Fisher Scientific, Waltham, MA, USA); ACQUITY-UPLCHSS-T3 column $(50 \times 1.0 \mathrm{~mm}$; Waters $)$ using an isocratic method and a methanol/water $(95 \% / 5 \%$, v/v) mobile phase with a flow rate of $300 \mu \mathrm{L} / \mathrm{min}$ and an injection volume of $3 \mu \mathrm{L}$. Acyclovir detection and quantification 
Fig. 2 Scheme (a) and photograph (b) of the duplicate test triad for comparative bioavailability assessment. Two treatment sites per test triad were dosed with the reference product $\left(R_{1}\right.$ : central, $R_{2}$ : noncentral) and one treatment site was dosed with the test product (T)
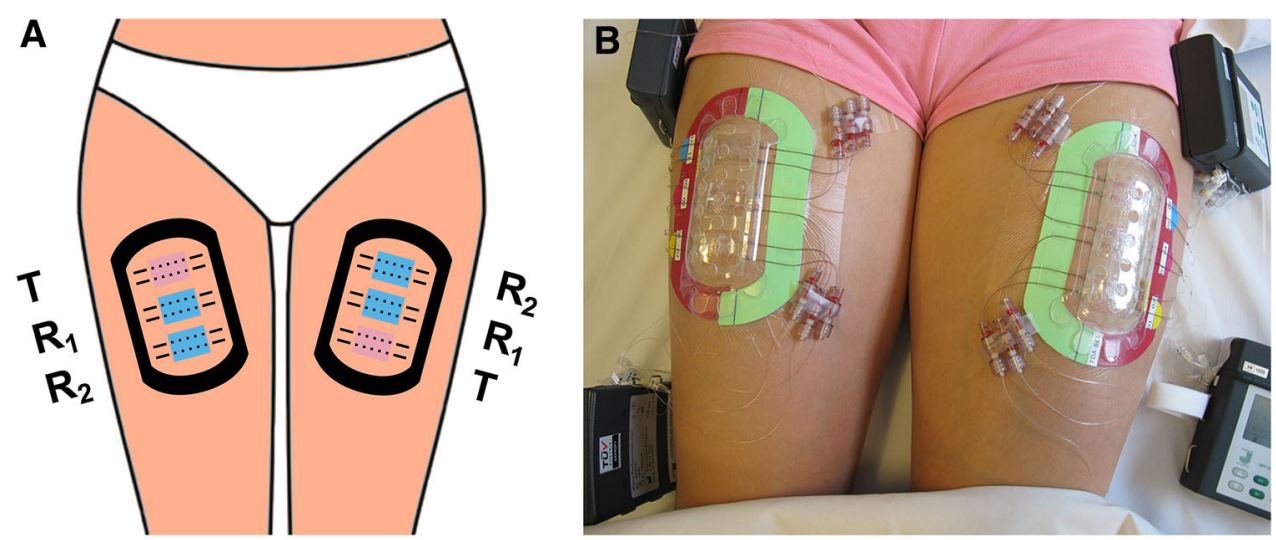

was performed by mass spectrometry (MS) using a Q-Exactive (Thermo) MS/MS in positive heated-electrospray mode $(\mathrm{m} / \mathrm{z} 226.0935-152.05635$ for acyclovir and 230.1196-152.05635 for the acyclovir-D4 internal standard).

\subsection{Pharmacokinetic Data Evaluation}

All clinical data were collected with an electronic case report form (eCRF) within OpenClinica Enterprise Edition. The eCRF system was hosted by Joanneum Research GmbH and is 21 CFR Part 11 compliant. All data management activities were performed and documented according to international standards and the data management plan.

The statistical analysis plan predefined data analysis, including criteria for the identification and treatment of possible outliers from intradermal 36-h concentration profiles. Possible outliers were identified by a statistical approach comparing each value with the moving median. Values that were $<50$ or $>200 \%$ of the moving median were reanalyzed and the reanalyzed values were used. Values that were still regarded as outliers according to this criterion were imputed by the arithmetic mean value of the preceding and subsequent time point samples, or by using the carry-last-value-forward approach in situations where there was no subsequent timepoint, to obtain complete profiles for PK endpoint calculation. The dermal PK endpoint $\mathrm{AUC}_{0-36 \mathrm{~h}}$ was calculated as sum of the $\mathrm{dOFM}$ sample concentrations over $36 \mathrm{~h}$ of continuous sampling. The dermal PK endpoint $C_{\max }$ was identified as the maximum concentration during the same duration.

An exploratory pilot study in six subjects where an even higher topical acyclovir dose was applied over $36 \mathrm{~h}$ showed no detectable acyclovir in the central test sites when the central test sites were left untreated, and it also did not show any detectable acyclovir in serum. Therefore, there was no evidence of any potential for cross-talk between test sites and all 40 test triads were considered independent for $\mathrm{BE}$ analysis in this proof-of-principle study.

$\mathrm{BE}$ criteria were used to evaluate the dermal PK endpoints for the positive control for $\mathrm{BE}\left(R_{2}\right.$ vs. $\left.R_{1}\right)$ and the negative control for $\mathrm{BE}$ ( $T$ vs. $R_{1}$ ) based on the typical acceptance criteria for BE. The dermal PK endpoints $\mathrm{AUC}_{0-36 \mathrm{~h}}$ and $C_{\max }$ were $\log$ transformed prior to analysis. The results were evaluated to determine whether the $90 \%$ confidence interval of the mean ratios of $T / R$ fell within the conventional BE limits of $0.80-1.25$.

\section{Results}

\section{1 dOFM Data Acquisition}

All subjects tolerated the 36-h continuous dOFM sampling and no dropouts or serious adverse events occurred. No adverse event (or serious adverse event) related to the dOFM technique occurred during the study. Dermal sampling and the probe perfusion equilibrium were stable for $36 \mathrm{~h}$, verified by stable glucose exchange rates (of approximately $60 \%$ ) across the probes. Applying the criterion for possible outliers within acyclovir profiles yielded a sample reanalysis rate of $3 \%$. After reanalysis, the rate of possible outliers was reduced to $1 \%$ of all samples. All the acyclovir profiles ( $36 \mathrm{~h}$ each) of the $240 \mathrm{dOFM}$ probes in the pivotal study (six probes per thigh on each of two thighs on each of 20 subjects) were included in the statistical evaluation. A characteristic steady decline in the later phase of the PK profile after $C_{\max }$, which is usually seen in systemic blood concentration PK studies, when the rate of drug clearance from the systemic circulation dominates over the rate of drug input into the systemic circulation (e.g., from an oral dose), was not clearly observed for acyclovir in the dermis after topical administration in this study, even across $36 \mathrm{~h}$ of sampling (Fig. 3). 


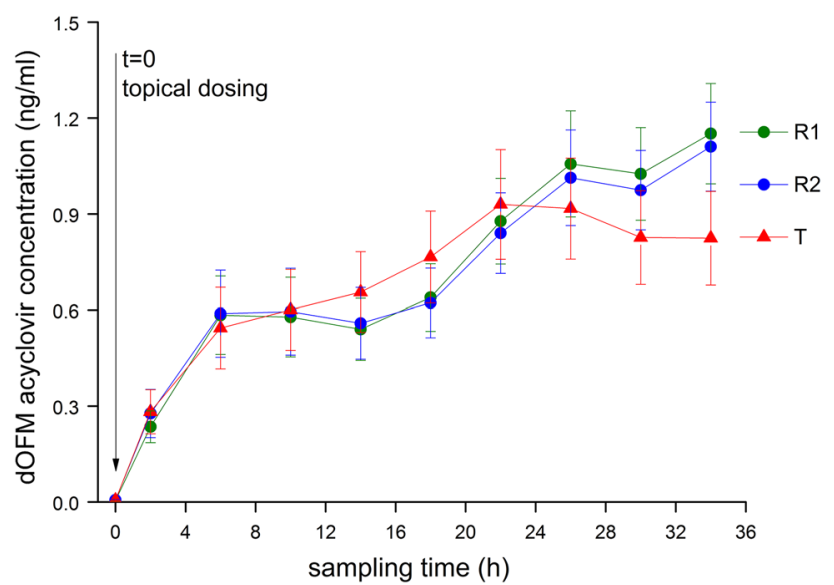

Fig. 3 Dermal open flow microperfusion (dOFM) acyclovir concentration profiles for the test product $(T)$ site and the two reference $\left(R_{1}\right.$ and $R_{2}$ ) sites (mean \pm standard error of the mean, $n=40$ test triads in 20 subjects). Acyclovir was analyzed from one pre-dose sample (spanning -1 to $0 \mathrm{~h}$ ) and nine pooled post-dose samples (spanning $0-4,4-8 \ldots 32-36 \mathrm{~h}$ ). The post-dose concentrations are plotted at the mid-point of the time intervals $(2,6 \ldots 34 \mathrm{~h})$

\subsection{Comparative BA}

The statistical comparison of PK endpoints using BE criteria are summarized in Table 1. The mean (and geometric mean) results across treatment groups for each PK endpoint are provided in Table 2 .

$\mathrm{BE}$ was confirmed for the positive control products $\left(\mathrm{R}_{2}\right.$ vs. $\left.\mathrm{R}_{1}\right)$ for $\mathrm{AUC}_{0-36 \mathrm{~h}}(0.86-1.18)$ and $C_{\max }(0.86-1.21)$. An exploratory statistical re-sampling procedure showed that $n=36$ (18 subjects in this study design) would have been sufficient to demonstrate $B E$ for $R_{2}$ vs. $R_{1}$ based on AUC $_{0-36 \mathrm{~h}}$ and $n=38$ (19 subjects in this study design) would have been sufficient based on $C_{\max }$.

The negative control products ( $T$ vs. $R_{1}$ ) failed to demonstrate $\mathrm{BE}$ for both parameters, $\mathrm{AUC}_{0-36 \mathrm{~h}}(0.69-1.05)$ and $C_{\max }(0.61-1.02)$.

Consistent probe depths were confirmed for the $R$ treatment sites $\left(R_{1}: 0.83 \pm 0.20 \mathrm{~mm}, R_{2}: 0.81 \pm 0.22 \mathrm{~mm}\right.$, $p=0.5329)$. The mean probe depth ( $T: 0.73 \pm 0.18 \mathrm{~mm}$, $p=0.0007$ ) was lower in the $T$ treatment sites than it was in either $R$ site, but a regression analysis indicated that there was no significant impact of probe depth on the AUC for acyclovir $(p=0.1001)$.

\section{Discussion}

This in vivo study illustrates the feasibility of dOFM to compare dermal PK and to assess BA of acyclovir from $T$ and $R$ topical creams in situ in the dermis. The results demonstrate that dOFM accurately and reproducibly determined the positive control products to be $\mathrm{BE}\left(\mathrm{R}_{2} \mathrm{vs}\right.$.
$\mathrm{R}_{1}$ ) and that it had the sensitivity to differentiate the negative control products ( $T$ vs. $R_{1}$ ) as not being $\mathrm{BE}$, in both cases based upon PK endpoints and the usual BE acceptance criteria.

The dOFM probes facilitated a stable intradermal sampling for $36 \mathrm{~h}$ from $4 R$ and $2 T$ treatment sites simultaneously in each subject, and thereby enabled characterization of the dermal PK profile for a relatively slow and low level of permeation of topical acyclovir. All subjects tolerated 36-h post-dose sampling well and no subject withdrew from the study prematurely. The positive subject compliance may be, in part, attributed to the compact wearable open-flow microperfusion devices, allowing enhanced mobility of the subjects. Bedside glucose analysis in dermal samples showed that exchange across the dOFM probe (the 'relative recovery') was stable over $36 \mathrm{~h}$ and thus indicated that skin cooling and the maintenance of an equilibrium period of $60 \mathrm{~min}$ post insertion was appropriate for this study.

dOFM delivered stable acyclovir profiles $(<1 \%$ outliers) and thereby demonstrated good reliability. Importantly, the high precision and reproducibility of the study results are attributable to several specific controls implemented to standardize and optimize study procedures, such as stabilization of the treatment sites to reduce strain and stretching, pain management during probe insertion to improve consistency and control of probe depth insertion, cooling after implementation to reduce trauma formation, and control of ambient temperature and humidity. Furthermore, several factors that were not necessarily amenable to better control, but which had a potential to influence the variability, were monitored so that their influence on variability could be evaluated and potentially corrected for: transepidermal water loss and impedance were monitored to evaluate potential subgroups in the population with skin permeability that may be more or less discriminating to differences in topical BA; glucose and deuterated water were monitored in the perfusate to identify any perturbation in the sampling environment; probe depth of the entire perfusate exchange area and flow rates of each time interval of each probe were monitored. The PK endpoint data in this study were not corrected by any of those factors.

Acyclovir products were chosen for this proof-of-principle dOFM study of comparative BA owing to the availability of $5 \%$ cream products with different compositions and potentially different BA, and also because a dermal PK approach may have particular value to assess BE of topical products intended for local action rather than a clinical endpoint study in patients. The hydrophilicity of this drug, however, is not representative of most topical drugs and further research with hydrophobic drugs is warranted. The $C_{\max }$ was not seen for the reference product within $36 \mathrm{~h}$, 
Table 1 Statistical evaluation comparing PK endpoints using typical BE criteria ( $n=40$ test triads in 20 subjects)

\begin{tabular}{lllll}
\hline Comparison & PK endpoint & $90 \%$ confidence interval & $T / R$ (point estimate) & Outcome \\
\hline$R_{2}$ vs. $R_{1}$ & $\mathrm{AUC}_{0-36 \mathrm{~h}}$ & $0.86-1.18$ & 1.01 & Positive BE result Confirmed \\
& $C_{\max }$ & $0.86-1.21$ & 1.02 & $R_{2}$ is considered BE to $R_{1}$ \\
$T$ vs. $R_{1}$ & $\mathrm{AUC}_{0-36 \mathrm{~h}}$ & $0.69-1.05$ & 0.85 & Negative BE result Confirmed \\
& $C_{\max }$ & $0.61-1.02$ & 0.79 & $T$ is not considered BE to $R_{1}$ \\
\hline
\end{tabular}

$A U C$ area under the curve, $B E$ bioequivalence, $C_{\max }$ maximum plasma concentration, $P K$ pharmacokinetic, $R$ reference, $T$ test product

Table 2 Pharmacokinetic endpoints $\mathrm{AUC}_{0-36 \mathrm{~h}}$ and $C_{\max }$

\begin{tabular}{|c|c|c|c|c|}
\hline \multirow[t]{2}{*}{ Product } & \multicolumn{2}{|c|}{$\mathrm{AUC}_{0-36 \mathrm{~h}}(\mathrm{ng} \mathrm{h} / \mathrm{mL})$} & \multicolumn{2}{|l|}{$C_{\max }(\mathrm{ng} / \mathrm{mL})$} \\
\hline & Mean \pm SEM $^{\mathrm{a}}$ & Geometric mean $\pm \mathrm{SEM}^{\mathrm{b}}$ & Mean \pm SEM & Geometric mean \pm SEM \\
\hline$R_{1}$ & $26.75 \pm 3.85$ & $16.23 \pm 1.19$ & $1.32 \pm 0.18$ & $0.85 \pm 1.18$ \\
\hline$R_{2}$ & $26.32 \pm 4.07$ & $16.34 \pm 1.18$ & $1.29 \pm 0.17$ & $0.86 \pm 1.17$ \\
\hline$T$ & $25.38 \pm 4.52$ & $13.84 \pm 1.20$ & $1.12 \pm 0.19$ & $0.67 \pm 1.19$ \\
\hline
\end{tabular}

$A U C$ area under the curve, $C_{\max }$ maximum plasma concentration, $R$ reference $S E M$ standard error of the mean, $T$ test product

${ }^{a}$ Mean refers to the arithmetic mean and corresponds to the arithmetic mean profiles in Fig. 2

${ }^{\mathrm{b}}$ Geometric mean refers to the geometric mean that was used in the statistical evaluation reported in Table 1 and the slow and ongoing permeation of acyclovir from both $5 \%$ cream products produced a $\mathrm{PK}$ profile from which it was not possible to calculate the $\mathrm{AUC}_{0-\infty}$. Hydrophilic drugs such as acyclovir may be poorly absorbed owing to a low partitioning through the skin's hydrophobic permeability barrier, the stratum corneum [26]. Low levels of percutaneous absorption and slow kinetics are among the reasons why prior studies using conventional dermal microdialysis, which were limited to a 5-h post-dose sampling duration, only succeeded to sample acyclovir when simultaneously inhibiting local skin blood flow [26, 27].

This study was intended to evaluate how accurately, precisely, and reproducibly dOFM could monitor the rate and extent to which a topically applied drug (acyclovir) becomes available in the dermis, and to compare the dermal BA of acyclovir between $T$ and $R$ creams administered using the same clinically relevant dose amount under essentially identical study conditions, using a single-dose study design. The labeled use of these acyclovir cream products involves a repeated dosing regimen throughout each day of use. Repeated dosing (e.g., every $4-5 \mathrm{~h}$ ) is an effective strategy to maintain therapeutic drug concentrations at the site of action. However, differences in BA that might not be evident in a study where the products were re-dosed every $4 \mathrm{~h}$ may become evident in clinical use, where the product may not be re-dosed precisely every $4 \mathrm{~h}$, particularly if one product dries more rapidly. In addition, a multiple dosing study design could obscure or convolute PK endpoints such as $C_{\max }$, which were of interest to compare. Therefore, a single-dose study design was considered to be the most appropriate for the purposes of comparing acyclovir BA between the $T$ and $R$ products precisely because it may be more sensitive and discriminating than a multiple dosing study design. Indeed, it is possible that the differences in BA observed between the $T$ and $R$ products in this study may not be distinguishable in the clinical use of these products.

The application of dOFM accurately and reproducibly confirmed BE when acyclovir cream $5 \%\left(\right.$ Zovirax $^{\circledR}$ ) was compared with itself $\left(\mathrm{R}_{2}\right.$ vs. $\left.\mathrm{R}_{1}\right)$. The statistical power estimation revealed that the sample size of $n=40$ (20 subjects in this study) was well chosen, as $n=38$ (19 subjects) would have been sufficient to demonstrate $\mathrm{BE}$ with a confidence level above $80 \%$. Both PK endpoints for the $T$ vs. $R$ product comparison failed to satisfy the conventional criteria for $\mathrm{BE}$, demonstrating that $\mathrm{dOFM}$ was sufficiently sensitive to discriminate between the $T$ and $R$ products.

\section{Conclusion}

In this study, dOFM showed low variability and high robustness, successfully characterizing the dermal BA of acyclovir from $T$ and $R$ products in a manner that could support evaluations of BE for topical acyclovir cream $5 \%$ 
products in vivo with $n=40$ (20 subjects in the independent replicate leg design used for this study). Factors contributing to variability of in vivo dermal PK data were well controlled in this clinical study, which is the first to evaluate clinical dOFM for comparative dermal $\mathrm{BA} / \mathrm{BE}$ assessment.

The results of this study suggest that an appropriately designed and well-controlled in vivo dOFM study could have the requisite accuracy, precision, reproducibility, and statistical power to compare the rate and extent to which a topically applied drug such as acyclovir becomes available in the dermis and that dOFM may provide a viable dermal PK approach for the BE assessment of topical drug products.

In the future, the sensitivity of dOFM may be even further improved for compounds permeating the skin more rapidly and to a greater extent than acyclovir, by refinements in subject inclusion/exclusion criteria, by correcting for perturbations to the dermal sampling caused by changes in blood flow or variations in probe depth, or by choosing shorter dose durations and monitoring the dermal PK for the $T$ and $R$ products during the period when the drug is being cleared from the skin. Further research exploring dOFM and other cutaneous PK methodologies to evaluate topical $\mathrm{BA} / \mathrm{BE}$ is warranted based upon the results of this study.

Acknowledgments The authors thank Alison Green for help writing the project proposal, Bernd Tschapeller and Christian Krainer for data management, Petra Baumann for statistics, Selma Mautner and Beate Boulgaropoulos for help in manuscript preparation (Joanneum Research GmbH, Graz, Austria), Sonja Kainz, Simon Schwingenschuh, Peter Reisenegger, Jürgen Lancaj, Joanna Adamczak, and Christian Höfferer (Joanneum Research $\mathrm{GmbH}$ ) and Stefanie SachFriedl, Eva Ekardt, Stefan Korsatko, Sarah Bischof, Robert Lipp, and Martina Brunner (Medical University, Graz, Austria) for their help with the clinical study, Denise Kollmann, Stefanie Weiss, and Anton Mautner (Joanneum Research $\mathrm{GmbH}$ ) for help with bioanalytics, Mike Roberts (University Brisbane, Australia) and Christopher Anderson (University of Linköping, Sweden) for the valuable discussions on potential covariates, Priyanka Ghosh (US Food and Drug Administraton) for scientific collaboration and project administration, and Elena Rantou and Youngsook Lee (US Food and Drug Administration) for their advice on BE statistics.

\section{Compliance with Ethical Standards}

Funding Funding for this project was made possible, in part, by the FDA through research award FD004946. The views expressed in this publication do not reflect the official policies of the FDA, or the Department of Health and Human Services; nor does any mention of trade names, commercial practices, or organization imply endorsement by the United States Government.

Conflict of interest MB, KIT, RR, BT, TA, CD, TB, SK, DS, TRP, and FS are employees of Joanneum Research holding patents on OFM devices. SGR is an employee of US FDA. GS and IK declare that there are no conflicts of interest.
Ethics, informed consent, research involving human participants The study was conducted with the full informed consent of all participating subjects, under the authority of the Ethical Committee of the Medical University of Graz, the Austrian health authority AGES, and the FDA's Research Involving Human Subjects Committee (RIHSC) and was performed in accordance with Good Clinical Practice and the ethical standards laid down in the 1964 Declaration of Helsinki and its later amendments. The study has been registered in the European Clinical Trials Register (EudraCT No. 2013-005062-19) and at ClinicalTrials.gov (NCT02711267).

Open Access This article is distributed under the terms of the Creative Commons Attribution-NonCommercial 4.0 International License (http://creativecommons.org/licenses/by-nc/4.0/), which permits any noncommercial use, distribution, and reproduction in any medium, provided you give appropriate credit to the original author(s) and the source, provide a link to the Creative Commons license, and indicate if changes were made.

\section{References}

1. Dunne S, Shannon B, Dunne C, Cullen W. A review of the differences and similarities between generic drugs and their originator counterparts, including economic benefits associated with usage of generic medicines, using Ireland as a case study. BMC Pharmacol Toxicol. 2013;14(1):1.

2. FDA. Guidance for industry: statistical approaches to establishing bioequivalence. Rockville: Center for Drug Evaluation and Research, Food and Drug Administration, FDA; 2001.

3. Schuirmann DJ. A comparison of the two one-sided tests procedure and the power approach for assessing the equivalence of average bioavailability. J Pharmacokinet Biopharm. 1987;15(6):657-80.

4. FDA. Guidance for industry: topical dermatological corticosteroids: in vivo bioequivalence, division of bioequivalence. Rockville: Center for Drug Evaluation and Research. Food and Drug Administration, FDA; 1995.

5. Kanfer I. Methods for the assessment of bioequivalence of topical dosage forms: correlations, optimization strategies and innovative approaches. In: Jenner J, Shah VP, Maibach HI, editors. Topical drug bioavailability, bioequivalence and penetration. 2nd ed. New York: Springer Science; 2015.

6. Lionberger RA. FDA critical path initiatives: opportunities for generic drug development. AAPS J. 2008;10(1):103-9.

7. Shah VP, Flynn GL, Yacobi A, et al. Bioequivalence of topical dermatological dosage forms: methods of evaluation of bioequivalence. AAPS/FDA workshop on bioequivalence of topical dermatological dosage forms: methods of evaluating bioequivalence. Skin Pharmacol Appl Skin Physiol. 1998;11(2):117-24.

8. Braddy AC, Davit BM, Stier EM, Conner DP. Survey of international regulatory bioequivalence recommendations for approval of generic topical dermatological drug products. AAPS J. 2015;17(1):121-33.

9. Chang R-K, Raw A, Lionberger R, Yu L. Generic development of topical dermatologic products: formulation development, process development, and testing of topical dermatologic products. AAPS J. 2013;15(1):41-52.

10. Kanfer I, Tettey-Amlalo RNO, Au WL, Hughes-Formella B. Assessment of topical dosage forms intended for local or regional activity. In: Shargel L, Kanfer I, editors. Generic drug product development: specialty drug products. New York and London: Informa Healthcare; 2010.

11. Raney SG, Franz TJ, Lehman PA, et al. Pharmacokinetics-based approaches for bioequivalence evaluation of topical 
dermatological drug products. Clin Pharmacokinet. 2015;54(11):1095-106.

12. Au WL, Skinner M, Kanfer I. Comparison of tape stripping with the human skin blanching assay for the bioequivalence assessment of topical clobetasol propionate formulations. J Pharm Pharm Sci. 2010;13(1):11-20.

13. Parfitt NR, Skinner MF, Bon C, Kanfer I. Bioequivalence of topical clotrimazole formulations: an improved tape stripping method. J Pharm Pharm Sci. 2011;14(3):347-57.

14. Kanfer I. Strategies for the bioequivalence assessment of topical dermatological dosage forms. J Bioequiv Availab. 2010;02(05): $102-10$.

15. Chaurasia CS, Müller M, Bashaw ED, et al. AAPS-FDA workshop white paper: microdialysis principles, application and regulatory perspectives. Pharm Res. 2007;24(5):1014-25.

16. Holmgaard R, Nielsen JB, Benfeldt E. Microdialysis sampling for investigations of bioavailability and bioequivalence of topically administered drugs: current state and future perspectives. Skin Pharmacol Physiol. 2010;23(5):225-43.

17. Herkenne C, Alberti I, Naik A, et al. In vivo methods for the assessment of topical drug bioavailability. Pharm Res. 2008;25(1):87-103.

18. Kreilgaard M. Assessment of cutaneous drug delivery using microdialysis. Adv Drug Deliv Rev. 2002;1(54 Suppl 1):S99-121.

19. Tettey-Amlalo RNO, Kanfer I, Skinner MF, et al. Application of dermal microdialysis for the evaluation of bioequivalence of a ketoprofen topical gel. Eur J Pharm Sci. 2009;36(2-3):219-25.

20. Au WL, Skinner MF, Benfeldt E, et al. Application of dermal microdialysis for the determination of bioavailability of clobetasol propionate applied to the skin of human subjects. Skin Pharmacol Physiol. 2012;25(1):17-24.

21. Benfeldt E, Hansen SH, Vølund A, et al. Bioequivalence of topical formulations in humans: evaluation by dermal microdialysis sampling and the dermatopharmacokinetic method. J Invest Dermatol. 2007;127(1):170-8.

22. Bodenlenz M, Höfferer C, Magnes C, et al. Dermal PK/PD of a lipophilic topical drug in psoriatic patients by continuous intradermal membrane-free sampling. Eur $\mathrm{J}$ Pharm Biopharm. 2012;81(3):635-41.

23. Bodenlenz M, Aigner B, Dragatin C, et al. Clinical applicability of dOFM devices for dermal sampling. Skin Res Technol. 2013;19(4):474-83.

24. Dragatin C, Polus F, Bodenlenz M, et al. Secukinumab distributes into dermal interstitial fluid of psoriasis patients as demonstrated by open flow microperfusion. Exp Dermatol. 2016;25(2):157-9.

25. Bodenlenz M, Dragatin C, Liebenberger L, et al. Penetration of clobetasol-17-propionate into psoriatic lesional and non-lesional skin assessed by dermal open flow microperfusion with time and space resolution. Pharm Res. 2016. [Epub ahead of print].

26. Morgan CJ, Renwick AG, Friedmann PS. The role of stratum corneum and dermal microvascular perfusion in penetration and tissue levels of water-soluble drugs investigated by microdialysis. Br J Dermatol. 2003;148(3):434-43.

27. Klimowicz A, Farfał S, Bielecka-Grzela S. Evaluation of skin penetration of topically applied drugs in humans by cutaneous microdialysis: acyclovir vs. salicylic acid. J Clin Pharm Ther. 2007;32(2):143-8. 